Gynäkologische Endokrinologie 2017·15:30-38 DOI 10.1007/s10304-016-0112-0

Online publiziert: 19. Januar 2017

(c) Der/die Autor(en) 2017. Dieser Artikel ist eine Open-Access-Publikation.

\section{Redaktion}

M. Birkhäuser, Basel

L. Wildt, Innsbruck

CrossMark

\section{Fuchs - K. Praxmarer · K. Sevecke}

Universitätsklinik für Kinder- und Jugendpsychiatrie, Department für Psychiatrie, Psychotherapie und Psychosomatik, Medizinische Universität Innsbruck, Innsbruck, Österreich

\title{
Transidentität in der Kinder- und Jugendpsychiatrie
}

\author{
Wissenschaftlicher Hintergrund und \\ klinische Erfahrungen aus einer \\ Spezialsprechstunde
}

\section{Fallbeispiel}

Die 15-jährige Christina („Jonas“; Namen für die Falldarstellung geändert) wird in Begleitung ihrer Eltern in der Transgendersprechstunde vorstellig. Sie sei von einer Beratungseinrichtung an unsere Klinik verwiesen worden. Christina (im Folgenden Jonas genannt) berichtet, dass sie seit der Pubertät und dem Einsetzen der Menses Gefühle einer massiven Unzufriedenheit mit sich selbst verspüre und die aufgrund ihres Körpers gesellschaftlich zugedachte Geschlechterrolle zunehmend ablehne. Diese Gefühle hätten anfangs verwirrt und großen Leidensdruck ausgelöst. Schon als Kind habe Christina rückblickend betrachtet immer mit Jungen gespielt und beispielsweise rosa Kleider "gehasst“. Die Eltern bestätigen das, in ihren Augen sei Christina immer schon „sehr burschikos“ aufgetreten. Jonas habe sich ab dem 13. Lebensjahr selbst verletzt, seine Eltern hätten dann auch eine Psychotherapie organisiert, in der er es aber nicht schaffte, „das entscheidende Thema" anzuschneiden.

Über Recherche im Internet und in sozialen Netzwerken sei ihm zunehmend klarer geworden, dass er ein "Transjunge“ sein könnte. Vor seinen Eltern und Geschwistern habe er aus Scham den Gedanken aber zunächst geheim gehalten und sich hauptsächlich mit gleichgesinnten Online-Bekanntschaften darüber ausgetauscht. Hierbei habe er zum ersten Mal das Gefühl verspürt, verstanden zu wer- den. Er habe dann den Tipp bekommen, eine Beratungseinrichtung für Jugendliche aufzusuchen. Dort habe man ihn hinsichtlich eines „Outings" im familiären Umfeld bestärkt und zu einem Kontakt mit der Klinik geraten. Jonas hat sehr konkrete Ziele: Er strebe eine „Hormontherapie“ an, zudem eine amtliche Personenstandsänderung. Dafür brauche er eine Bestätigung von der Klinik.

\section{Einleitung}

Geschlechtsdysphorische Kinder und Jugendliche wie Jonas sind zunehmend Thema im öffentlichen Diskurs, sowohl in Europa [1-3] als auch in den USA, wo beispielsweise die angesehene New York Times seit 2015 einen eigenen Blograum für Erfahrungsberichte von transidenten Menschen zur Verfügung stellt [4].

Auch im wissenschaftlichen Bereich bezeugen steigende Publikationszahlen die immer stärkere Auseinandersetzung mit geschlechtsdysphorischen jungen Menschen: So wurden unter der Verschlagwortung „transgender“ und „youth“ im Jahr 2000 vier einschlägige Studien veröffentlicht, im Jahr 2014 war diese Zahl bereits auf 145 angestiegen [5].

Vor dem Hintergrund dieser Entwicklung sind Beratungseinrichtungen und spezialisierte Kliniken zunehmend mit gut vernetzten und informierten Kindern, Jugendlichen und Familien konfrontiert, die sehr genaue Erwartungen an eine mögliche Behandlung haben [6]. Unverändert fehlen aber verbindliche Richtlinien, die für Kinder und Jugendliche $\mathrm{zu}$ einheitlichen Behandlungsentscheidungen führen, nicht zuletzt, weil das Phänomen transidenter junger Menschen auch unter Fachleuten kontrovers bewertet wird [7-11]. Durch das breite Spektrum unterschiedlicher ideologischer Zugänge entsteht für kinder- und jugendpsychiatrische Spezialeinrichtungen der Auftrag, als zentrale Drehscheibe in der Behandlung von transidenten jungen Menschen $\mathrm{zu}$ fungieren und möglichst neutral und evidenzbezogen die Risiken und Chancen möglicher Therapieschritte zu vermitteln.

\section{》) Das Phänomen transidenter junger Menschen wird auch unter Fachleuten kontrovers bewertet}

In diesem Beitrag sollen daher überblicksmäßig die derzeit gebräuchliche Terminologie und Klassifikation sowie verfügbare wissenschaftliche Evidenz zur Behandlung von transidenten Kindern und Jugendlichen dargestellt werden. Ergänzend werden die ersten klinischen Erfahrungen einer neuen universitären Spezialambulanz geschildert. Abschließend möchten wir aus unserer Sicht wichtige Kernaussagen in einem Fazit zusammenfassen. 


\section{Tab. 1 Diagnostische Kriterien der Geschlechtsdysphorie im Kindesalter nach DSM-5 [12]}

A) Eine seit mindestens 6 Monaten bestehende ausgeprägte Diskrepanz zwischen Gender und Zuweisungsgeschlecht, wobei mindestens 6 der folgenden Kriterien erfüllt sein müssen (Kriterium 1 obligat)

1. Ausgeprägtes Verlangen oder Insistieren, dem anderen Geschlecht (oder einem alternativen Gender, das sich vom Zuweisungsgeschlecht unterscheidet) anzugehören

2. Bei Kindern mit männlichem Zuweisungsgeschlecht: ausgeprägte Vorliebe, sich weiblich zu kleiden und zu schminken; bei Kindern mit weiblichem Zuweisungsgeschlecht: ausgeprägte Vorliebe für ausschließlich typisch maskuline Kleidung und großer Widerstand, typisch feminine Kleidung zu tragen

3. Ausgeprägte Vorliebe dafür, in Rollen- und Fantasiespielen gegengeschlechtliche Rollen einzunehmen

4. Ausgeprägte Vorliebe für Spielzeug, Spiele oder Aktivitäten, mit denen sich Kinder des anderen Geschlechts typischerweise beschäftigen

5. Ausgeprägte Vorliebe für Spielgefährten des anderen Geschlechts

6. Kinder mit männlichem Zuweisungsgeschlecht: ausgeprägte Ablehnung von typisch jungenhaften Spielen, Spielzeug und Aktivitäten sowie ausgeprägte Vermeidung von Raufen und Balgen; Kinder mit weiblichem Zuweisungsgeschlecht: ausgeprägte Ablehnung von typisch mädchenhaften Spielen, Spielzeug und Freizeitaktivitäten

7. Ausgeprägte Ablehnung der eigenen primären Geschlechtsmerkmale

8. Ausgeprägtes Verlangen nach den primären und/oder sekundären Geschlechtsmerkmalen im Einklang mit dem erlebten Gender

B) Klinisch bedeutsames Leiden oder Beeinträchtigung in sozialen, schulischen oder anderen wichtigen Funktionsbereichen

Tab. 2 Diagnostische Kriterien der Geschlechtsdysphorie im Jugend- und Erwachsenenalter nach DSM-5 [12]

A) Eine seit mindestens 6 Monaten bestehende ausgeprägte Diskrepanz zwischen Gender und Zuweisungsgeschlecht, wobei mindestens 2 der folgenden Kriterien erfüllt sein müssen

1. Ausgeprägte Diskrepanz zwischen Gender und den primären und/oder sekundären Geschlechtsmerkmalen (oder bei Jugendlichen: den erwarteten sekundären Geschlechtsmerkmalen)

2. Ausgeprägtes Verlangen, die eigenen primären und/oder sekundären Geschlechtsmerkmale loszuwerden (oder bei Jugendlichen: das Verlangen, die Entwicklung der erwarteten sekundären Geschlechtsmerkmale zu verhindern)

3. Ausgeprägtes Verlangen nach den primären und/oder sekundären Geschlechtsmerkmalen des anderen Geschlechts

4. Ausgeprägtes Verlangen, dem anderen Geschlecht anzugehören (oder einem alternativen Gender, das sich vom Zuweisungsgeschlecht unterscheidet)

5. Ausgeprägtes Verlangen danach, wie das andere Geschlecht behandelt zu werden (oder wie ein alternatives Gender, das sich vom Zuweisungsgeschlecht unterscheidet)

6. Ausgeprägte Überzeugung, die typischen Gefühle und Reaktionsweisen des anderen Geschlechts aufzuweisen (oder die eines alternativen Genders, das sich vom Zuweisungsgeschlecht unterscheidet)

B) Klinisch bedeutsames Leiden oder Beeinträchtigung in sozialen, schulischen oder anderen wichtigen Funktionsbereichen

\section{Klassifikation und Terminologie}

Mit Einführung des Begriffs Geschlechtsdysphorie im Diagnostischen und Statistischen Manual psychischer Störungen DSM-5 [12] wurden die zuvor gebräuchlichen Ausdrücke Transsexualität und Geschlechtsidentitätsstörung abgelöst, zugleich wurde der Wandel im gesellschaftlichen Umgang mit Geschlechtlichkeit in einem offiziellen Diagnosemanual verankert. Geschlechtsdysphorie (GD) bezeichnet laut DSM-5 einen klinisch relevanten Leidensdruck, der sich aus der Diskrepanz zwischen Gender und dem üblicherweise bei der Geburt festgelegten Zuweisungsgeschlecht ergibt.

Der Begriff Gender steht laut den Autoren für die öffentliche und in der Regel gesetzlich anerkannte Rolle als
Junge oder Mädchen bzw. Mann oder Frau. Im Manual wird der Genderbegriff als etwas biologisch und psychosozial determiniertes angesehen. Damit wird eher eine Gegenposition zu konstruktivistischen Gendertheorien eingenommen. Unter Gender wird aber etwas völlig anderes verstanden als unter dem Begriff Geschlecht, der sich auf „biologische Merkmale zur Unterscheidung von männlich und weiblich im Sinne der Reproduktionsfähigkeit" bezieht. Zuweisungsgeschlecht bzw. Geschlechtszuweisung nimmt auf den Vorgang Bezug, einem Neugeborenen anhand anatomischer primärer Geschlechtsmerkmale ein weibliches oder ein männliches Geschlecht zuzuweisen. Der Begriff Transgender schließlich bezieht sich auf Personen, „die sich vorübergehend oder dauernd mit einem Gender identifizieren, das sich von ihrem Zuweisungsgeschlecht unterscheidet".

Laut den Autoren des DSM-5 soll mit dem Label GD ein deskriptiver und weniger wertender Begriff eingeführt werden, der das klinische Problem der Dysphorie in den Mittelpunkt stellt. Dadurch kann vermieden werden, die Identität eines Kindes oder Jugendlichen als etwas Pathologisches oder zu Behandelndes anzusprechen, wie das mit früheren Bezeichnungen wie Geschlechtsidentitätsstörung der Fall war [13].

Eine Behandlung von GD soll laut DSM-5 Geschlechtsangleichung genannt werden, darunter sollte die Summe aus medizinischen Interventionen und der offiziellen Personenstandsänderung verstanden werden. Die Geschlechtsangleichung hat zum Ziel, die individuelle Geschlechtsidentität $\mathrm{zu}$ erreichen, worunter die subjektive und individuelle „Identifikation einer Person als männlich, weiblich oder einer weiteren Kategorie" verstanden wird.

Während im ersten Teil der neuen Kriterien zunächst ein dichotomer Genderbegriff angeführt wird („JungeMädchen“, „Mann-Frau“) wird in einem späteren Abschnitt ausdrücklich betont, dass Gender auch „alternative Geschlechtsidentitäten" beinhalten kann und sich die GD nicht notwendigerweise „darauf beschränken muss, dem anderen Geschlecht anzugehören“. 
Diagnostische Kriterien für Kinder bzw. für Jugendliche und Erwachsene (- Tab. 1 und 2) unterscheiden sich in ihrer Bezugnahme auf alterstypische Verhaltensweisen, werden aber im DSM-5 in einem einheitlichen Kapitel verortet.

Nach Reisner et al. [14] hat sich verschiedentlich auch die Konvention durchgesetzt, sowohl Zuweisungsgeschlecht als auch Gender explizit zu nennen: Transfeminine Personen oder Mann-zu-Frau-Personen („male to female“ [MTF]) wären hierbei biologisch männliche Menschen mit einem weiblichen Gender, transmaskuline Personen oder Frau-zu-Mann-Personen („female to male" $[\mathrm{FTM}]$ ) biologisch weibliche Menschen mit einem männlichen Gender.

\section{》) Die ICD-10 beschreibt Geschlechtsdysphorie im juvenilen Alter als psychische Erkrankung}

Als nicht mehr zeitgemäß wird von den meisten Fachleuten die Terminologie der Internationalen statistischen Klassifikation der Krankheiten (ICD-10) angesehen, die von einer „Störung der Geschlechtsidentität des Kindesalters (F64.2)“ bzw. von „Transsexualismus (F64.0)“ spricht und diese Erkrankungen in verschiedenen Kapiteln platziert. Beide Störungen sind im Abschnitt „F6: Persönlichkeitsund Verhaltensstörungen“ verortet [15]. Damit wird das Erleben von Geschlechtsdysphorie in der juvenilen Altersperiode als eine psychische Erkrankung beschrieben, die sich von einer objektiv richtigen Identität abhebt. Manche Autoren sehen darin eine Analogie zur „Diagnose Homosexualität" in früheren Ausgaben der ICD [6]. Bezüglich der für 2017 erwarteten ICD-11 wird daher diskutiert, diese beiden Begriffe aufzulösen, sie durch die Diagnose "gender incongruence“ zu ersetzen und die diagnostischen Kategorien für Kinder, Jugendliche und Erwachsene in einem einheitlichen Kapitel zu verorten. Der Ausdruck „gender incongruence" soll die Unstimmigkeit zwischen Gender und Zuweisungsgeschlecht ausdrücken [16].

Gynäkologische Endokrinologie 2017 · 15:30-38 DOI 10.1007/s10304-016-0112-0

(c) Der/die Autor(en) 2017. Dieser Artikel ist eine Open-Access-Publikation.

\section{Fuchs $\cdot$ K. Praxmarer $\cdot$ K. Sevecke}

Transidentität in der Kinder- und Jugendpsychiatrie. Wissenschaftlicher Hintergrund und klinische Erfahrungen aus einer Spezialsprechstunde

\section{Zusammenfassung}

Hintergrund. Transidentität bei Jugendlichen ist zunehmend ein Thema im öffentlichen Diskurs und auch ein möglicher Behandlungsauftrag in spezialisierten Beratungseinrichtungen.

Ziel der Arbeit. Ziel der Arbeit ist deshalb eine überblicksmäßige Darstellung der derzeit gebräuchlichen Terminologie und Klassifikation sowie der verfügbaren wissenschaftlichen Evidenz und erhältlichen Leitlinien aus kinder- und jugendpsychiatrischer Sicht. Ergänzend sollen klinische Erfahrungen aus den ersten eineinhalb Jahren einer neu errichteten Spezialsprechstunde für jugendliche Transidentität reflektiert werden. Material und Methoden. Auswertung offizieller Diagnosemanuale (ICD-10, DSM-5), Darstellung von Leitlinien, Überblick über klinische Studien, Fallberichte eigener Patienten.

Ergebnisse. Begrüßenswerte entstigmatisierende Klassifikationsmöglichkeiten in Diagnosemanualen sowie eine Reihe von
Leitlinien und klinischen Studien stellen einen zunehmend robusteren Boden für ein evidenzbasiertes Vorgehen bei jugendlicher Transidentität dar. Dennoch ist die praktische Umsetzung dieser Erkenntnisse in eine konkrete Behandlungsentscheidung weit von Einheitlichkeit entfernt.

Schlussfolgerungen. Vor diesem Hintergrund muss eine kinder- und jugendpsychiatrische Spezialsprechstunde aus unserer Sicht eine möglichst wertneutrale Haltung einnehmen und den jungen Menschen wie auch ihren Familien Entfaltungspotenzial sowie Zugang zu Behandlungsmöglichkeiten bieten. Teil dieser Grundhaltung muss aber auch sein, mit der gebotenen Sorgfalt auf mögliche Schwierigkeiten im Langzeitverlauf hinzuweisen.

Schlüsselwörter

Klassifikation/Transgender - Terminologie . Behandlung/Transgender - Leitlinien . Geschlechtsdysphorie

\section{Transgender identity in pediatric and adolescent psychiatry. Scientific background and clinical experiences from a special counseling facility}

\section{Abstract}

Background. Transgender identity in youth has increasingly become a topic of public discourse and a potential treatment issue for specialized counseling facilities.

Aim. The goal of this article is to provide an overview from the perspective of pediatric and adolescent psychiatry of the following: a) current terminology and classification and $b$ ) available scientific evidence and guidelines. A further goal was to reflect on the past 1.5 years of clinical experiences with outpatient services for youth with transgender identity.

Material and methods. Evaluation of official diagnostic manuals (ICD-10, DSM-5), discussion of clinical studies, guidelines and case studies of our patients.

Results. Destigmatized classification options in official manuals are a welcome development as are a number of clinical studies and guidelines that provide a robust foundation for evidence-based approaches when dealing with youth with transgender identity; however, the practical implementation of the results in concrete treatment measures is far from consistent.

Conclusion. From our experience, specialized outpatient services provided by pediatric and adolescent psychiatric facilities should take as non-judgmental a stance as possible, providing youth and their families avenues for development as well as access to treatment. A part of this stance should also be to carefully point out the potential long-term difficulties with the necessary caution.

\section{Keywords}

Classification/transgender persons . Terminology as topic - Treatment/transgender persons - Guidelines as topic - Gender dysphoria 


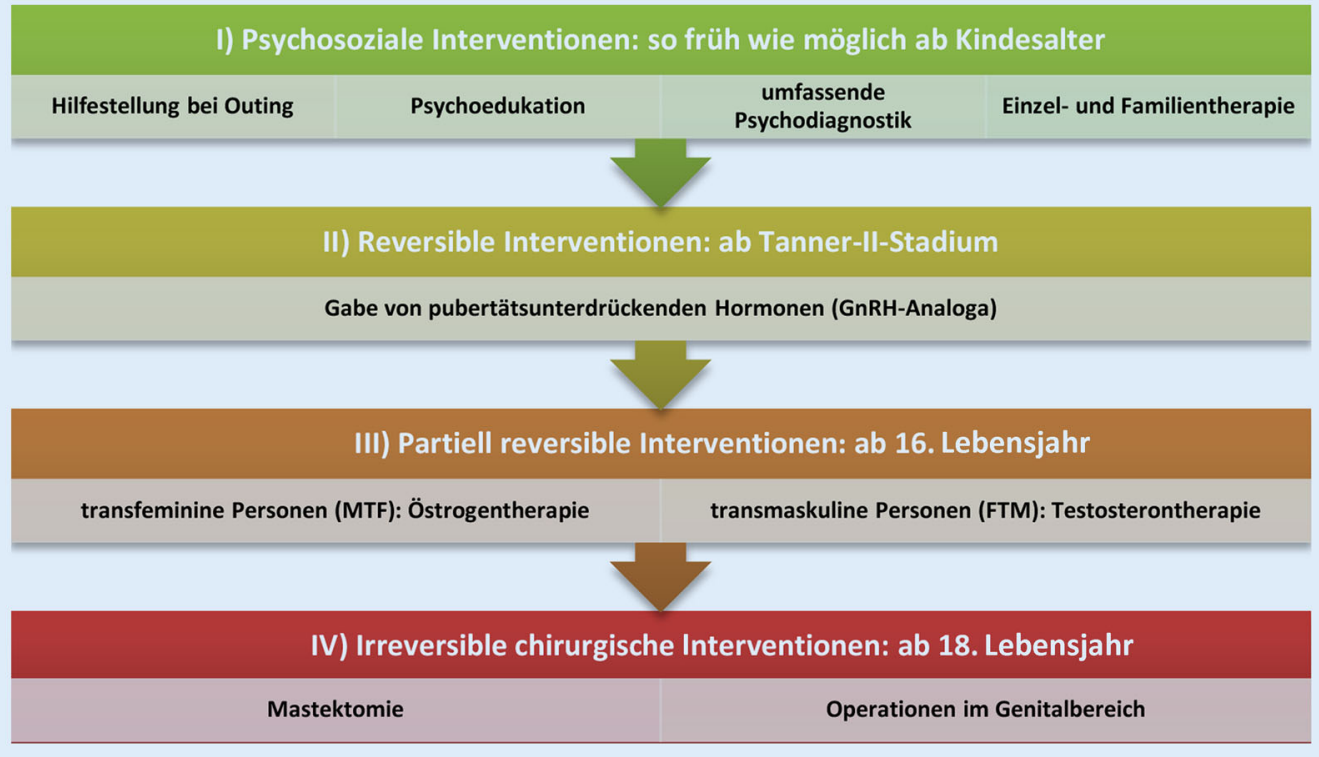

Abb. $1 \varangle$ Abfolge von Behandlungsschritten. FTM "Female to male" (Frau zu Mann); GnRH Gonadotropin-Releasing-Hormon; MTF "male to female" (Mann zu Frau). (Nach [17-21])

Im klinischen Alltag in Österreich sind Ärzte zum Gebrauch von Diagnosen nach ICD-10 verpflichtet. Bei im Behandlungsprozess stehenden jungen Menschen muss daher meist die Diagnose „Transsexualismus (F64.0)“verwendet werden.

\section{Verfügbare Leitlinien zur Behandlung transidenter Jugendlicher}

Wichtige Leitlinien zur Behandlung geschlechtsdysphorischer junger Menschen sind auf internationaler Ebene die "Standards of care for the health of transsexual, transgender, and gender-nonconforming people, version $7^{\text {“ }}$ der World Professional Association for Transgender Health (WPATH; [17]) sowie die klinische Leitlinie „Endocrine treatment of transsexual persons" der Endocrine Society [18]. Beide Leitlinien enthalten in Unterkapiteln jeweils Empfehlungen für Kinder und Jugendliche. In einer weiteren Publikation veröffentlichte eine Taskforce im Auftrag der American Psychiatric Association (APA) 2012 eine ausführliche Literaturübersicht mit häufiger Bezugnahme auf minderjährige Patienten [19].

Auf deutschsprachiger Ebene wurden 2013 die AWMF-Leitlinien „Störungen der Geschlechtsidentität im Kindes- und Jugendalter (F64)“ der Deutschen Gesell- schaft für Kinder- und Jugendpsychiatrie, Psychosomatik und Psychotherapie (DGKJP) veröffentlicht [20]. Österreichische Leitlinien zur Behandlung transidenter junger Menschen wurden von einer interdisziplinären Arbeitsgruppe, der auch der Autor dieses Beitrags angehört, 2016 fertiggestellt und zur Publikation im offiziellen Organ der Österreichischen Gesellschaft für Kinderund Jugendpsychiatrie, Psychosomatik und Psychotherapie (ÖGKJP) eingereicht [21]. Die Leitlinien wurden dem Bundesministerium für Gesundheit und Frauen vorgelegt.

Zusammenfassend empfehlen die verfügbaren Leitlinien ähnliche Eckpunkte bzw. eine Abfolge von Behandlungsschritten, der sich auch die österreichischen Empfehlungen angeschlossen haben. Grundlegend werden als Basis jeglicher Behandlung folgende Schritte empfohlen, die möglichst früh durchgeführt werden sollten [19]:

- Ausführliche psychodiagnostische Abklärung der GD

- Ausführliche psychodiagnostische Abklärung vorhandener komorbider Störungen, im Bedarfsfall Behandlung dieser Störungen

- Analyse der familiären Ressourcen, Identifizierung von problematischen Interaktionen auf Familienebene und (so vorhanden) Ansprechen dieser Probleme
- Aufklärung der Sorgeberechtigten über alle potenziell möglichen Behandlungsoptionen, Ansprechen der limitierten Evidenzlage, Ansprechen möglicher Langzeitverläufe

- Altersgerechte Aufklärung des Kindes selbst

- Analyse der Umgebungsfaktoren (Schule, Familie, soziales Umfeld) auf mögliche protektive, aber auch auf destabilisierende Faktoren wie mögliches Mobbing oder mögliche Stigmatisierung, bei Bedarf Angebot von Hilfsmaßnahmen

Alle Leitlinien sind sich derzeitig einig, eine pubertätsunterdrückende Therapie nicht vor Einsetzen der Pubertät zu beginnen, als Kriterium werden eine $\mathrm{Pu}$ bertätsentwicklung bis mindestens zum Tanner-2-Stadium sowie eine sich daraus deutlich entwickelnde GD gesehen. Weitere Einigkeit besteht in der Empfehlung, ab einem Alter von 16 Jahren als nächsten möglichen Schritt eine nur mehr partiell reversible Therapie mittels gegengeschlechtlicher Hormone zu ermöglichen. Irreversible (chirurgische) Interventionen werden $\mathrm{ab}$ dem 18 . Geburtstag (rechtliche Volljährigkeit) empfohlen.

In $\triangle A b b .1$ ist der mögliche leitliniengerechte Behandlungsrahmen zusammengefasst. 


\section{Infobox 1 Leitlinien zur Be- handlung transidenter Kinder und Jugendlicher}

- World Professional Association for Transgender Health (WPATH): „Standards of care for the health of transsexual, transgender, and gender-nonconforming people, version 7" [17]

- Endocrine Society: „Endocrine treatment of transsexual persons: an Endocrine Society clinical practice guideline" [18]

- Leitlinien der Arbeitsgemeinschaft Wissenschaftlicher Medizinischer Fachgesellschaften (AWMF) und der Deutschen Gesellschaft für Kinder- und Jugendpsychiatrie, Psychosomatik und Psychotherapie (DGKJP): „Störungen der Geschlechtsidentität im Kindes- und Jugendalter (F64)" [20]

- Österreichische Empfehlungen für den Behandlungsprozess bei Geschlechtsdysphorie von Kindern und Jugendlichen, in Vorbereitung [21]

Für eine genauere Darstellung der somatischen Aspekte der GonadotropinReleasing-Hormon-Analoga-Therapie sowie der gegengeschlechtlichen Hormontherapie bei Jugendlichen wird auf den Beitrag von Kapelari in dieser Ausgabe von Gynäkologische Endokrinologie verwiesen.

\section{Entwicklungsepidemiologie}

Bisher steht weltweit lediglich eine Studie mit einer repräsentativen bevölkerungsbasierten Stichprobe zur Verfügung, in der die Prävalenz von Transidentität bei Jugendlichen untersucht wurde. In dieser Studie mit über 8000 Teilnehmern aus Neuseeland wurde unter anderem die Frage gestellt, ob der Jugendliche glaubt, Transgender zu sein, in einer Anmerkung wurde dieser Begriff näher erklärt. Ein Anteil von 1,2 \% der befragten Jugendlichen antwortete, Transgender $\mathrm{zu}$ sein, 2,5\% gaben an, sich bezüglich ihres Genders nicht sicher zu sein [22]. Eine Prävalenzrate von $1,2 \%$ unter Jugendlichen würde allerdings eine weitaus höhere Prävalenzrate im Kindesalter bedeuten: Die Mehrzahl aller Kinder, die vor der Pubertät eine GD erleben, arrangiert sich im Laufe der Zeit mit diesen Gefühlen. Je nach Studie erleben lediglich 2-27\% aller Kinder, die wegen GD vorstellig wurden, diese Gefühle auch als Jugendliche und möchten sich deshalb behandeln lassen. Anders formuliert liegt der Prozentsatz derjenigen Jugendlichen, deren GD-Erleben in der Adoleszenz nach Einsetzen der Pubertät nahezu sistierte, in Studien bei knapp $85 \%$ [23].

Entwicklungsverläufe von Kindern, die vor der Pubertät eine ausgeprägte GD erleben, lassen sich in 3 Gruppen unterteilen $[23,24]$ :

- „Desisting gender dysphoria“ („Desister") - Gruppe A: Kinder arrangieren sich mit ihren geschlechtsspezifischen Körpermerkmalen und entwickeln später in der Pubertät eine homosexuelle oder bisexuelle Orientierung $\rightarrow$ häufigster Verlauf!

- „Desisting gender dysphoria“ („Desister") - Gruppe B: Kinder arrangieren sich mit ihren geschlechtsspezifischen Körpermerkmalen und entwickeln später in der Pubertät eine ausschließlich heterosexuelle Orientierung $\rightarrow$ seltenster Verlauf!

- „Persisting gender dysphoria“ („Persister"): Die GD persistiert bis in die beginnende Adoleszenz und exazerbiert im Zuge der in der Pubertät beginnenden Entwicklung der sekundären Geschlechtsmerkmale $\rightarrow$ Behandlungswunsch und möglicherweise Behandlungsindikation

Möller et al. [24] betonen, dass einer möglichst zuverlässigen Identifikation der „Persister" eine Schlüsselrolle im Behandlungsprozess von GD bei Kindern und Jugendlichen zukommt: Es gilt, „Desister" nicht vorschnell mit einer nicht indizierten und möglicherweise irreversiblen Hormonbehandlung zu belasten, umgekehrt aber eindeutige „Persister" auf ihrem Weg optimal zu unterstützen und ihnen auch eine pubertätsunterdrückende und das gewünschte Geschlecht induzierende Behandlung zu ermöglichen.

Die wenigen verfügbaren Longitudinaldaten weisen darauf hin, dass „Persister" im Kindesalter folgende Merkmale aufweisen [23]:

- Starke Ausprägung der GD

- Weibliches Geschlecht

- Homosexuelle Orientierung
- Stärkere Sichtbarkeit geschlechtsrollennonkonformer Verhaltensweisen

- Überzeugung, das andere Geschlecht zu sein („Ich bin ein Junge“), wohingegen „Desister“ eher wie das andere Geschlecht sein wollen („Ich wäre gern wie ein Junge“)

\section{Behandlung transidenter Jugendlicher}

Zentrale Fragen hinsichtlich einer möglichen Behandlung von GD im Kindesund Jugendalter sind nach Möller et al. [24]:

- Ethische Fragestellung: Ist ein irreversibler Eingriff in eine natürlich ablaufende geschlechtliche Entwicklung gerechtfertigt?

- Diagnostische Fragestellung: Wie kann man möglichst gut die Gruppe junger Menschen identifizieren, in der eine Unterdrückung der ursprünglichen körperlichen Pubertätsentwicklung sowie ein künstliches Induzieren gewünschter sekundärer Geschlechtsmerkmale indiziert sind („Persister“)?

- Ist eine Einschätzung möglich, ob die GD mit einer Psychotherapie bereits ausreichend behandelt ist und ob keine körperlichen Interventionen nötig sind?

- Frage des richtigen Zeitpunkts: Ab wann sollte welche Behandlung erfolgen?

Die publizierten Längsschnittstudien zeigen für Jugendliche, die leitlinienkonform (- Infobox 1) zunächst mittels Pubertätssuppression und später mittels Induktion gewünschter sekundärer Geschlechtsmerkmale behandelt wurden, erfreuliche Ergebnisse. Entsprechend hat sich dieses vor 15 Jahren eingeführte Protokoll („Dutch model“) in den meisten Behandlungszentren durchgesetzt [25]. In einer aktuellen niederländischen Studie wurde eine Gruppe von Jugendlichen $\mathrm{zu}$ Beginn der $\mathrm{Pu}$ bertätssuppression (Durchschnittsalter 13,6 Jahre), zu Beginn der gegengeschlechtlichen Hormontherapie (Durchschnittsalter 16,7 Jahre) und im jungen Erwachsenenalter (Durchschnittsalter 20,7 Jahre) ausführlich psychodiagnos- 
Hier steht eine Anzeige.

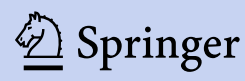




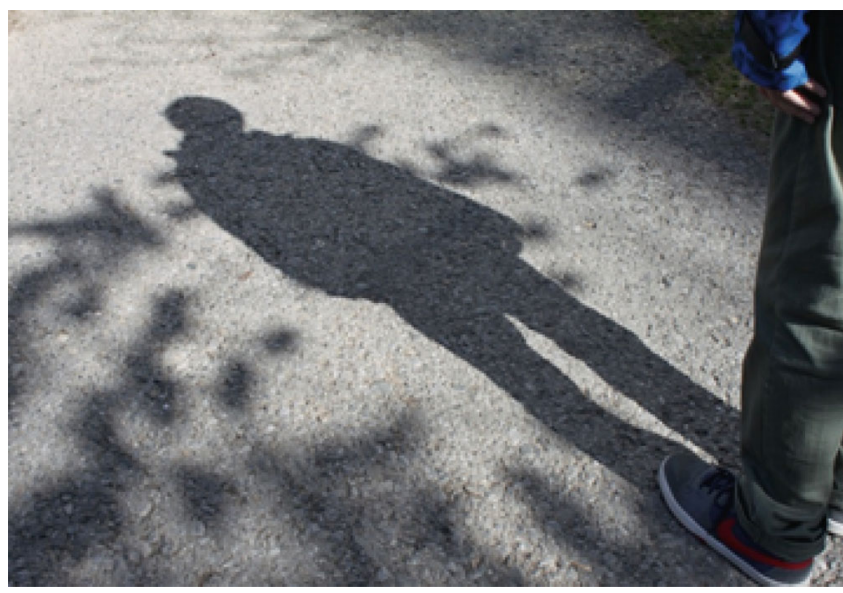

Abb. $2 \Delta$ In der Kunsttherapie wurde der Schatten einer Patientin (Frau zu Mann) fotografiert und damit über soziale Rollenbilder reflektiert. (Mit freundl. Genehmigung von Barbara Oberhofer, Österreich)

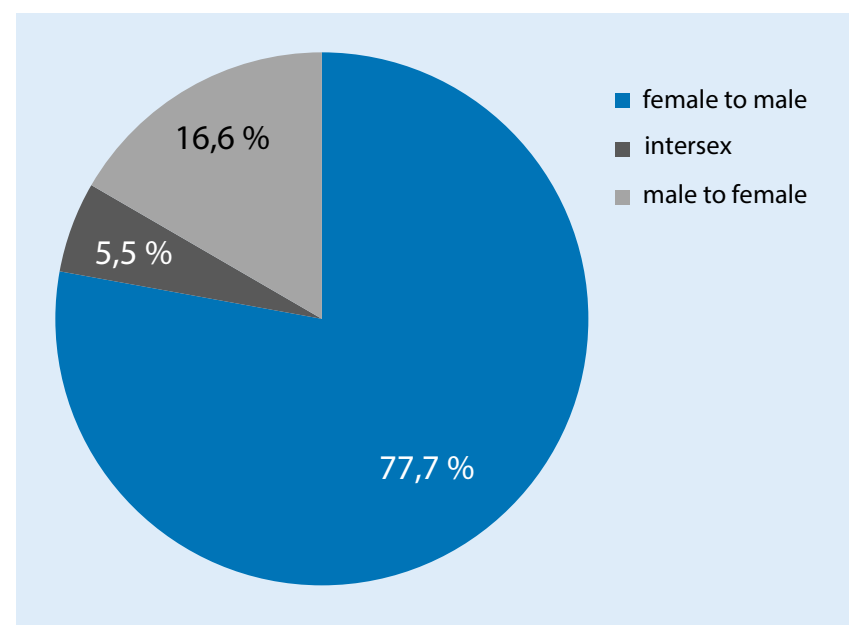

Abb. $3 \Delta$ Diagnostische Verteilung bisheriger Patienten in der Transgenderambulanz $(n=19)$ tisch untersucht. Hier zeigte sich eine gute longitudinale Entwicklung. Die Patienten wiesen nach der stufenweisen Behandlung keine GD mehr auf, ihr subjektives Wohlbefinden und das psychosoziale Funktionsniveau unterschieden sich nicht von einer gesunden Kontrollgruppe [26]. Im bisher längsten dokumentierten Verlaufsfall zeigten sich bei einem FTM-Patienten, der mit 13 Jahren eine pubertätsunterdrückende Therapie sowie ab einem Alter von 17 Jahren androgene Hormone bekommen hatte, nach einer 22-jährigen Beobachtungsperiode keinerlei somatische Komplikationen [27].

Eine aktuelle Studie zeigte bezüglich der Behandlung von Kindern mit GD, dass auch bei sehr jungen Patienten ein Leben in der gegengeschlechtlichen Rolle unter laufender professioneller psychologischer Beratung zu einer deutlichen Reduktion der psychopathologischen Symptombelastung führen kann. Die in die Studie eingeschlossenen Kinder (Durchschnittsalter 7,7 Jahre) wurden in der sozialen Transition von ihren Familien ausdrücklich unterstützt und wiesen in den untersuchten Bereichen Angst und Depression keine höheren Werte auf als eine Kontrollgruppe [28].

Junge Menschen mit geschlechtsdysphorischem Erleben zeigten bisher im Vergleich $\mathrm{zu}$ heterosexuellen Kindern und Jugendlichen ohne GD ein deutlich schlechteres psychisches Wohlbefinden mit erhöhten Raten von affektiven
Störungen, Angststörungen, Selbstverletzungen und Suizidgedanken. Studien weisen darauf hin, dass diese Probleme vor allem durch sozialen, aber auch durch intrapsychischen Druck ausgelöst werden, der aus der Unmöglichkeit entsteht, eine gewünschte Identität zu leben. Soziale Akzeptanz bzw. ein Coming-out konnten als schützende Faktoren identifiziert werden [29].

\section{》) Junge Menschen mit unbe- handelter Geschlechtsdysphorie zeigen ein deutlich schlechteres psychisches Wohlbefinden}

Zusammenfassend weist also eine immer robustere Datenlage darauf hin, dass eine leitliniengerechte Behandlung von transidenten Kindern und Jugendlichen angesichts einer deutlich erhöhten psychopathologischen Symptombelastung klar indiziert ist. Die derzeit angewandten Behandlungsprotokolle können in dieser Patientengruppe zu einer Konsolidierung führen.

\section{Eigene Erfahrungen nach eineinhalb Jahren Transgenderambulanz}

Zunehmende Konsultationen von transidenten Kindern, Jugendlichen (• Abb. 2) und deren Familien haben uns dazu bewogen, an der Universitätsklinik für Kinder- und Jugendpsychiatrie in Innsbruck eine interdisziplinäre Transgendersprechstunde einzurichten. Als Anbieter von tertiären Gesundheitsleistungen verfügen die Universitätskliniken bzw. die Medizinische Universität Innsbruck über ein geeignetes Netz aus spezialisierten Einrichtungen. Somit konnten wir 2014 in Zusammenarbeit mit der Universitätsklinik für Gynäkologische Endokrinologie sowie dem Department für Kinder- und Jugendheilkunde Innsbruck (Spezialbereich Endokrinologie) unsere Sprechstunde starten.

Wir sehen unser Angebot als einen notwendigen Schritt, um einen leitliniengerechten und interdisziplinären Kompetenzbereich zu ermöglichen. Dazu haben wir neben der Erstellung eines internen Ablaufstandards auch den Rahmen eines „Transgenderboards“ für Kinder und Jugendliche geschaffen, in dem Fallbesprechungen erfolgen und Behandlungsentscheidungen getroffen werden. Die Einführung einer Selbsthilfegruppe für betroffene Eltern wurde von uns forciert und hat sich, neben der engen Zusammenarbeit mit einschlägigen extramuralen Beratungseinrichtungen, bewährt.

Im Einklang mit internationalen Trends [30] waren unsere bisherigen Patienten in der überwiegenden Mehrzahl transmaskuline Jugendliche, also biologisch weibliche Menschen mit männlichem Gender (• Abb. 3). Bei allen Patienten hatte zum Zeitpunkt der Erstvorstellung die Pubertät bereits be- 
gonnen, sie waren somit „Persister“ und erfüllten die Leitlinienkriterien für den Beginn einer pubertätsunterdrückenden Therapie. Bis auf eine Patientin mit intersexuellem Gender, die den Wunsch nach Psychotherapie formulierte, bestand bei allen Patienten ein eindeutiger Behandlungs- und Transitionswunsch.

In unserer kinder-und jugendpsychiatrischen Spezialsprechstunde übernehmen ein Arzt, ein Sozialarbeiter sowie eine klinische Psychologin und Psychotherapeutin

- das Erstgespräch,

- die Psychodiagnostik,

- die Vernetzung mit den Sorgeberechtigten,

- die Sozialberatung,

- die ausführliche Psychoedukation,

- die Vermittlung einer Psychotherapie,

- die Vernetzung mit dem extramuralen Helfersystem und

- die Vernetzung mit den intramuralen somatischen Behandlern.

Behandlungsentscheidungen sind trotz Bezugnahme auf Leitlinien immer Einzelfallentscheidungen und werden ausschließlich im multiprofessionellen Team getroffen. Zunächst erfolgen eine umfangreiche Diagnostikund Verlaufsbeobachtung (diese ist für Jugendliche, die unsere Sprechstunde bereits mit viel Druck und genauen Erwartungen an eine Behandlung aufsuchen, häufig schwer auszuhalten). Danach wird gemeinsam mit der Familie ein individueller Behandlungsplan erstellt.

\section{Fazit für die Praxis}

- Nur wenige Kinder, die wegen GD vorstellig werden, erleben diese Gefühle auch als Jugendliche und möchten sich deshalb behandeln lassen.

- Hält die GD jedoch bis in die Pubertät an, kann dies zu einer ernsthaften psychopathologischen Symptombelastung bis hin zu Suizidalität führen. In diesem Fall ist eine eindeutige Behandlungsindikation gegeben.

- Längsschnittstudien zeigen für Kinder und Jugendliche, die leitlinienge- recht behandelt werden, erfreuliche Ergebnisse.

- Ein vor 15 Jahren eingeführtes Behandlungsprotokoll („Dutch model“) hat sich in den meisten Behandlungszentren durchgesetzt.

- Nur ein interdisziplinäres und entwicklungsbegleitendes Vorgehen kann der individuellen Situation der jungen Patienten und ihrer Familien Rechnung tragen.

- Der Beitrag der Kinder- und Jugendpsychiatrie ist dabei auch eine sorgfältige psychiatrische Differenzialdiagnostik. Es gilt, im Jugendalter Störungen wie Psychosen oder beginnende Persönlichkeitsstörungen auszuschließen und behandelbare komorbide Störungen zu erkennen und zu therapieren.

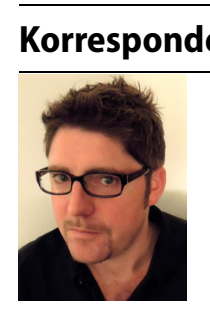

\section{Dr. M. Fuchs}

Universitätsklinik für Kinderund Jugendpsychiatrie, Department für Psychiatrie, Psychotherapie und Psychosomatik, Medizinische Universität Innsbruck Anichstr. 35, 6020 Innsbruck, Österreich martin.fuchs@tirol-kliniken.at

Danksagung. Die Autoren bedanken sich bei Barbara Oberhofer für das Überlassen von Abb. 2. Open access funding provided by University of Innsbruck and Medical University of Innsbruck.

\section{Einhaltung ethischer Richtlinien}

Interessenkonflikt. M. Fuchs, K. Praxmarer und K. Sevecke geben an, dass kein Interessenkonflikt besteht.

Dieser Beitrag beinhaltet keine von den Autoren durchgeführten Studien an Menschen oder Tieren.

Open Access Dieser Artikel wird unter der Creative Commons Namensnennung 4.0 International Lizenz (http://creativecommons.org/licenses/by/4.0/deed. de) veröffentlicht, welche die Nutzung, Vervielfältigung, Bearbeitung, Verbreitung und Wiedergabe in jeglichem Medium und Format erlaubt, sofern Sie den/die ursprünglichen Autor(en) und die Quelle ordnungsgemäßnennen, einen Link zur Creative Commons Lizenz beifügen und angeben, ob Änderungen vorgenommen wurden.

\section{Literatur}

1. Barthélémy A (2016) Transgender-Kinder: In mir drin bin ich ein Mädchen. http://derstandard. at/2000031595271/Transgender-Kinder-In-mirdrin-bin-ich-ein-Maedchen. Zugegriffen: 23. Nov. 2016

2. Gaul S (2016) Transidente Kinder: Nie wieder Paul sein. http://www.zeit.de/gesellschaft/ zeitgeschehen/2016-05/transidente-kindertrans-maedchen-forschung. Zugegriffen: 23. Nov. 2016

3. Spiewak M (2016) Transsexualität: Das ist kein Spleen. http://www.zeit.de/2016/47/ transsexualitaet-kinder-jugendliche-geschlechtumwandlung. Zugegriffen:28. Nov. 2016

4. n.a. (2016) Transgender Lives: Your Stories. http://www.nytimes.com/interactive/projects/ storywall/transgender-today. Zugegriffen: 23.11. 2016

5. n.a. (2016) Transgender youth. https://www.ncbi. nlm.nih.gov/pubmed/?term=transgender+youth Zugegriffen:29. Nov. 2016

6. Moeller B, Romer G (2014) Geschlechtsdysphorie im Kindes- und Jugendalter. Prax Kinderpsychol Kinderpsychiatr 63(6):431-436

7. Rutzen KM, Nieder TO, Schreier H, Möller B (2014) Die Versorgung von Kindern und Jugendlichen mit Geschlechtsdysphorie aus der Sicht internationaler Expertise. Prax Kinderpsychol Kinderpsychiatr 63(6):449-464

8. Vance SR Jr, Ehrensaft D, Rosenthal SM (2014) Psychological and medical care of gender nonconforming youth. Pediatrics 134(6):1184-1192

9. Olson J, Schrager SM, Belzer M, Simons LK, Clark LF (2015) Baseline physiologic and psychosocial characteristics of transgender youth seeking care for gender dysphoria. J Adolesc Health 57(4):374-380

10. Trumbull D, Cretella MA, Grossman M (2015) Puberty is not a disorder. Pediatrics 135(5):e1366

11. Vance SR Jr, Ehrensaft D, Rosenthal SM (2015) Author's response. Pediatrics 135(5):e1366-e1367

12. American Psychiatric Association (2013) DSM5. Diagnostic and statistical manual of mental disorders, 5. Aufl. APA, Arlington

13. Schneider C, Cerwenka S, Nieder TO, Briken P, Cohen-Kettenis PT, De Cuypere G, Haraldsen IR, Kreukels BP, Richter-Appelt H (2016) Measuring gender dysphoria: a multicenter examination and comparison of the Utrecht gender dysphoria scale and the gender identity/gender dysphoria questionnaire for adolescents and adults. Arch Sex Behav 45(3):551-558

14. Reisner SL, Poteat T, Keatley J, Cabral M, Mothopeng T, Dunham E, Holland CE, Max R, Baral SD (2016) Global health burden and needs of transgender populations: a review. Lancet 388(10042):412-436

15. (2012) F6: Persönlichkeits- und Verhaltensstörungen. In: Dilling $\mathrm{H}$, Freyberger $\mathrm{HJ}$ (Hrsg) Taschenführer zur ICD-10-Klassifikation psychischer Störungen: nach dem Pocket Guide von J.E. Cooper. Hans Huber Hogrefe, Bern, S231-272

16. Winter S, De Cuypere G, Green J, Kane R, Knudson G (2016) The Proposed ICD-11 Gender Incongruence of Childhood Diagnosis: A World Professional Association for Transgender Health Membership Survey. Arch Sex Behav 45(7):1605-1614

17. Coleman E, Bockting W, Botzer M, Cohen-Kettenis P, DeCuypere G, Feldman J, Fraser L, Green J, Knudson G, MeyerWJ, MonstreyS, Adler RK, Brown GR, Devor AH, Ehrbar R, Ettner R, EylerE, Garofalo R, Karasic DH, Lev Al, Mayer G, Meyer-Bahlburg H, Hall BP, Pfaefflin F, Rachlin K, Robinson B, Schechter LS, 
Tangpricha V, van Trotsenburg M, Vitale A, Winter S, Whittle S, Wylie KR, Zucker K (2012) Standards of care for the health of transsexual, transgender, and gender-nonconforming people, version 7.Int J Transgenderism 13(4):165-232

18. Hembree WC, Cohen-Kettenis $P$, Delemarre-van de Waal HA, Gooren LJ, Meyer WJ 3rd, Spack NP, Tangpricha V, Montori VM (2009) Endocrine treatment of transsexual persons: an Endocrine Society clinical practice guideline.JClin Endocrinol Metab 94(9):3132-3154

19. Byne W, Bradley SJ, Coleman E, Eyler AE, Green R, Menvielle EJ, Meyer-Bahlburg HF, Pleak RR, Tompkins DA, American Psychiatric Association Task Force on Treatment of Gender Identity Disorder (2012) Report of the American Psychiatric Association Task Force on Treatment of Gender Identity Disorder. Arch Sex Behav 41(4):759-796

20. Meyenburg B, Korte A, Möller B, Romer G (2013) AWMF-Leitlinien Störungen der Geschlechtsidentität im Kindes- und Jugendalter (F64) - Deutsche Gesellschaft für Kinder- und Jugendpsychiatrie, Psychosomatik und Psychotherapie. http:// www.awmf.org/uploads/tx_szleitlinien/028014I_S1_Störungen_Geschlechtsidentität_201308_01.pdf.Zugegriffen:23. Nov. 2016

21. Thun-Hohenstein $L$, Hackenberg B, Riedl $S$, Bangerl HP, Fuchs M, Gottardi-Butturini E (2016) Empfehlungen für den Behandlungsprozess bei Geschlechtsdysphorie von Kindern und Jugendlichen. neuropsychiatrie (eingereicht)

22. Clark TC, Lucassen MF, Bullen P, Denny SJ, Fleming TM, Robinson EM, Rossen FV (2014) The health and well-being of transgender high school students: results from the New Zealand adolescent health survey (Youth'12). J Adolesc Health 55(1):93-99

23. SteensmaTD, McGuire JK, Kreukels BP, Beekman AJ, Cohen-Kettenis PT (2013) Factors associated with desistence and persistence of childhood gender dysphoria: a quantitative follow-up study. J Am Acad Child Adolesc Psychiatry 52(6):582-590

24. Möller B, NiederTO, Preuss WF, Beckerl, Fahrenkrug S, Wüsthof A, Briken P, Romer G, Richter-Appelt H (2014) Versorgung von Kindern und Jugendlichen mit Geschlechtsdysphorie im Rahmen einer interdisziplinären Spezialsprechstunde. Prax Kinderpsychol Kinderpsychiatr 63(6):465-485

25. Cohen-Kettenis PT, Delemarre-van de Waal HA, Gooren LJ (2008) The treatment of adolescent transsexuals: changing insights. J Sex Med 5(8):1892-1897

26. de Vries AL, McGuire JK, Steensma TD, Wagenaar EC, Doreleijers TA, Cohen-Kettenis PT (2014) Young adult psychological outcome after puberty suppression and gender reassignment. Pediatrics 134(4):696-704

27. Cohen-Kettenis PT, Schagen SE, Steensma TD, de Vries AL, Delemarre-van deWaal HA (2011) Puberty suppression in a gender-dysphoric adolescent: a 22-yearfollow-up. Arch Sex Behav 40(4):843-847

28. Olson KR, Durwood L, DeMeules M, McLaughlin KA (2016) Mental health of transgender children who are supported in their identities. Pediatrics 137(3):e20153223

29. Russell ST, Fish JN (2016) Mental health in lesbian, gay, bisexual, and transgender (LGBT) youth. Annu Rev Clin Psychol 12:465-487

30. Aitken $M$, Steensma TD, Blanchard R, VanderLaan DP, Wood H, Fuentes A, Spegg C, Wasserman L, Ames M, Fitzsimmons CL, Leef JH, Lishak V, Reim E, Takagi A, Vinik J, Wreford J, Cohen-Kettenis PT, de Vries AL, Kreukels BP, Zucker KJ (2015) Evidence for an altered sex ratio in clinic-referred adolescents with gender dysphoria.J SexMed 12:756-763

\section{SpringerMedizin.de Lesen Sie Ihre Fachzeitschrift auch als ePaper!}

Als Abonnent können Sie Ihre Zeitschrift in verschiedenen Formaten lesen. Wählen Sie je nach Vorliebe und Situation aus, ob Sie die Zeitschrift als Print-Ausgabe, in Form von einzelnen Beiträgen auf springermedizin.de oder aber als komplette, elektronische ePaper-Ausgabe lesen möchten.

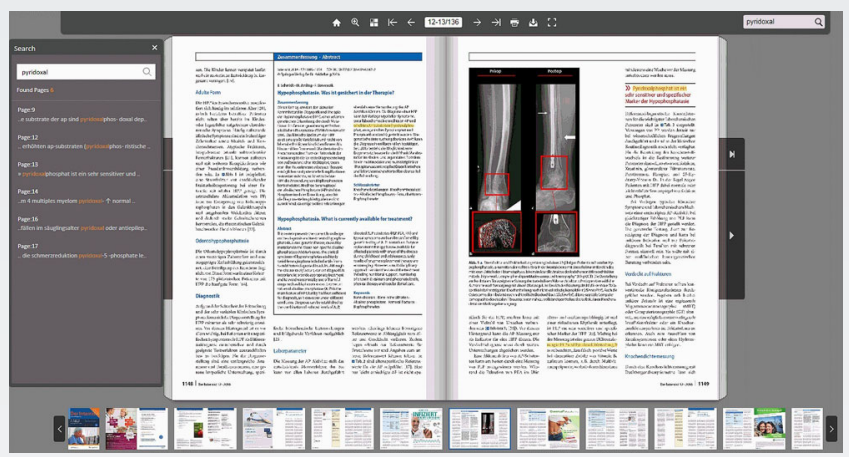

Die ePaper sind die identische Form der gedruckten Ausgaben. Sie sind nutzbar auf verschiedenen Endgeräten wie PC, Tablet oder Smartphone

Das sind die Vorteile des ePapers:
$>$ Das verlinkte Inhaltsverzeichnis führt Sie direkt zum gewünschten Beitrag
$>$ Eine Suchfunktion ermöglicht das Auffinden von Schlagworten innerhalb der Zeitschrift.
> Jede Ausgabe kann als PDF heruntergeladen und damit auch offline gelesen werden bzw. auch gespeichert oder ausgedruckt werden.
> Als Abonnent haben Sie Zugang zu allen ePaper-Ausgaben ab 2016.

Sie finden die ePaper auf SpringerMedizin.de bei der jeweiligen Ausgabe Ihrer Fachzeitschrift. Klicken Sie auf den Button „Zum ePaper”. 